\title{
Pourrais-je avoir des lignes directrices pour m'aider à interpréter les lignes directrices thérapeutiques?
}

\author{
par James E Tisdale
}

$\int_{\text {Qu' }}^{\prime}$ 'ai ai besoin d'aide. Je suis encore plus perplexe que d'habitude, ce que mes amis et collègues n'auraient jamais cru possible. Parce que plusieurs séries de lignes directrices sur le traitement des maladies cardiovasculaires ont été publiées récemment, et que certaines se contredisent.

Comme bon nombre de cliniciens, je m'appuie sur des lignes directrices thérapeutiques fondées sur des données probantes afin de choisir le meilleur traitement pour mes patients. Ces derniers mois, plusieurs nouvelles lignes directrices conseillant les cliniciens sur les traitements destinés aux patients atteints de différentes maladies cardiovasculaires (mon domaine d'expertise) ont été publiées. Ce devrait être une bonne nouvelle. Toutefois, il y a aussi de mauvaises nouvelles, c'est-à-dire qu'il y a plusieurs lignes directrices sur des sujets identiques (particulièrement sur l'hypertension et la réduction du risque cardiovasculaire) et que bon nombre d'entre elles offrent des recommandations divergentes, voire contradictoires. Plutôt que de m'éclairer sur le traitement à adopter pour les maladies cardiovasculaires, ces nouvelles lignes directrices ont obscurci mon jugement.

Dans la chronique "Le pour et le contre " de ce numéro du JCPH, Loewen ${ }^{1}$ et Pharand $^{2}$ débattent de la pertinence des lignes directrices communes du American College of Cardiology (ACC) et de l'American Heart Association (AHA) sur l'utilisation des hypolipidémiants ${ }^{3}$ et ils se demandent s'il faut ou non en encourager l'utilisation. Les deux auteurs relèvent la nature controversée de ces lignes directrices de même que le fait qu'elles different grandement de celles de la Société canadienne de cardiologie pour diagnostiquer et traiter la dyslipidémie afin de prévenir les maladies cardiovasculaires ${ }^{4}$, qui, elles, ont été publiées seulement quelques mois avant les lignes directrices communes du ACC et de l'AHA. D'une certaine façon, les nouvelles lignes directrices sur le cholestérol (que l'on peut décrire de manière générale comme des lignes directrices sur la réduction du risque cardiovasculaire) sont mieux fondées sur des données probantes que les précédentes, car elles reconnaissent qu'il n'y a pas assez de preuves des bienfaits d'atteindre des taux précis de cholestérol à lipoprotéines de basse densité (LDL). De plus, on souligne que les données probantes qui existent montrent surtout que les statines sont efficaces pour diminuer la morbidité et la mortalité liées aux maladies cardiovasculaires. Toutefois, l'abandon des valeurs cibles précises de cholestérol LDL, comme le recommandent les lignes directrices communes du ACC et de l'AHA, est controversé. En outre, les niveaux de risque cardiovasculaire à partir desquels on recommande les statines sont quelque peu arbitraires et le seuil de traitement serait trop bas pour la prévention primaires. De plus, le calculateur de risque fourni pour mettre en pratique les lignes directrices pourrait surestimer le risque, menant ainsi à un traitement excessif des patients ${ }^{6}$. Donc, parmi ces séries de lignes directrices, laquelle est la bonne et comment les cliniciens peuvent-ils déterminer quelle est la meilleure façon de traiter leurs patients?

Les diverses séries de lignes directrices de prise en charge de l'hypertension publiées ces derniers mois ont aussi brouillé les pistes, à tout le moins pour moi. Celles du Eighth Joint National Committee (JNC 8) ${ }^{7}$, publiées plus tôt cette année, établissaient une nouvelle cible tensionnelle de traitement $(<150 / 90 \mathrm{~mm}$ $\mathrm{Hg}$ ) pour les patients de 60 ans et plus. Pourtant, cette cible de traitement pour cette population de patients (au moins ceux qui ont jusqu'à 80 ans) est différente de celle (< 140/90 mm Hg) recommandée dans les lignes directrices du traitement de l'hypertension qui sont promulguées par l'American Society for Hypertension et l'International Society of Hypertension ${ }^{8}$, publiées environ au même moment que celles du JNC 8. De plus, certaines recommandations pharmacothérapeutiques de ces lignes directrices divergent, notamment celles portant sur la pharmacothérapie initiale pour les patients qui ne sont pas de race noire.

Compte tenu de ces différences (qui ne sont pas les seules) entre les recommandations des diverses lignes directrices sur l'hypertension, la diminution du risque et le cholestérol, 
comment un clinicien peut-il prendre une décision? Le caractère contradictoire et controversé d'un bon nombre de lignes directrices thérapeutiques permet de faire ressortir les lacunes dans les données probantes et de mettre en évidence la nécessité d'effectuer plus de recherches afin de pouvoir orienter les gens vers la meilleure méthode de diagnostic et le meilleur traitement. Mais repérer des lacunes dans les données probantes disponibles, des lacunes auxquelles de futures recherches devraient s'attaquer, n'aide pas les cliniciens à choisir dès aujourd'hui un traitement pour un patient. Krumholz ${ }^{9}$ croit que " les lignes directrices devraient informer et non dicter, guider et non imposer, soutenir et non restreindre " [traduction libre]. Il a aussi souligné que, lorsqu'ils appliquent les lignes directrices thérapeutiques, les cliniciens ne devraient pas imposer des choix aux patients; ils devraient plutôt collaborer avec eux en leur offrant assez d'information fondée sur des données probantes dans le but de les aider à faire leur propre choix quant au meilleur traitement à adopter pour leur maladie?. Cette approche dépasse la simple mise en application des recommandations de lignes directrices afin d'inclure les choix, préférences, valeurs et buts des patients de même que les considérations propres à chaque patient quant à la qualité de vie. Ceci permet donc aux patients de prendre part au processus décisionnel concernant leur traitement.

Mais la question demeure : comment les cliniciens peuvent-ils interpréter différentes lignes directrices dont les recommandations divergent et tout de même émettre des recommandations thérapeutiques adéquates aux patients, ou au moins donner aux patients les meilleures preuves pour leur permettre de faire leur propre choix de traitement? Les lignes directrices thérapeutiques mêmes présentent les fondements, les données probantes et les explications motivant les recommandations qu'elles contiennent, et il peut être nécessaire pour les cliniciens d'étudier et de digérer ces informations. Peut-être devront-ils consulter certaines des données probantes d'origine citées dans les lignes directrices thérapeutiques afin de se forger une opinion éclairée des données et recommandations. De plus, les cliniciens peuvent évaluer la crédibilité des lignes directrices encadrant le traitement clinique à l'aide des critères élaborés par l'Institute of Medicine ${ }^{10}$. Lorsque les cliniciens appliquent ces huit normes à des lignes directrices thérapeutiques, ils profitent d'un moyen objectif pour évaluer la crédibilité des recommandations. La méthode GRADE (Grading of Recommendations Assessment, Development and Evaluation) ${ }^{11}$ offre aussi un cadre permettant à ceux qui utilisent des lignes directrices d'évaluer la qualité des données probantes et la force des recommandations.

L'utilisation de ces approches permettra-t-elle de dissiper la confusion qui règne chez moi et chez d'autres cliniciens, nous qui sommes incertains des moyens à prendre pour réconcilier les recommandations diagnostiques et thérapeutiques différentes énoncées dans diverses lignes directrices sur une même maladie? Pas complètement. Mais elles peuvent au moins aider les cliniciens à offrir les meilleures preuves et informations à leurs patients pour qu'ainsi l'équipe patient-clinicien puisse arriver à choisir le meilleur plan de traitement.

Références

1. Loewen P. Are the new guidelines for the use of lipid-lowering agents sound, and should their adoption be encouraged? The "pro" side. Can J Hosp Pharm. 2014;67(3):246-7.

2. Pharand C. Are the new guidelines for the use of lipid-lowering agents sound, and should their adoption be encouraged? The "con" side. Can J Hosp Pharm. 2014;67(3):247-9.

3. Stone NJ, Robinson J, Lichtenstein AH, Bairey Mertz CN, Blum CB, Eckel $\mathrm{RH}$, et coll. 2013 ACC/AHA guideline on the treatment of blood cholesterol to reduce atherosclerotic cardiovascular risk in adults: a report of the American College of Cardiology/American Heart Association Task Force on Practice Guidelines. Circulation. 12 novembre 2013 (diffusion en ligne avant impression). DOI 10.1161/01.cir.0000437738.63853.7a/

4. Anderson TJ, Grégoire J, Hegele RA, Couture P, Mancini GB, McPherson R, et coll. 2012 update of the Canadian Cardiovascular Society guidelines for the diagnosis and treatment of dyslipidemia for the prevention of cardiovascular disease in the adult. Can J Cardiol. 2013;29(2):151-67.

5. Downs J, Good C. New cholesterol guidelines; has Godot finally arrived? Ann Intern Med. 2014;160(5):354-6.

6. Ridker PM, Cook NR. Statins: new American guidelines for prevention of cardiovascular disease. Lancet. 2013;382(9907):1762-5.

7. James PA, Oparil S, Carter BL, Cushman WC, Dennison-Himmelfarb C, Handler J, et coll. 2014 evidence-based guideline for the management of high blood pressure in adults: report from the panel members appointed to the Eighth Joint National Committee (JNC 8). JAMA. 2014;311(5):507-20.

8. Weber MA, Schiffrin EL, White WB, Mann S, Lindholm LH, Kenerson JG, et coll. Clinical practice guidelines for the management of hypertension in the community: a statement by the American Society of Hypertension and the International Society of Hypertension. J Clin Hypertens. 2014;16(1):14-26.

9. Krumholz HM. The new cholesterol and blood pressure guidelines: perspective on the path forward [éditorial]. JAMA. 2014;311(14):1403-5.

10. Graham R, Mancher M, Miller Woman D, Greenfield S, Steinberg E, rédacteurs; Institute of Medicine, Committee on Standards for Developing Trustworthy Clinical Practice Guidelines. Clinical practice guidelines we can trust. Washington (DC) : National Academies Press; 2011.

11. Atkins D, Best D, Briss PA, Eccles M, Falck-Ytter Y, Flottorp S, et coll. Grading quality of evidence and strength of recommendations. BMJ. 2004;328(7454):1490.

James E Tisdale, B. Sc. Pharm., Pharm. D, est professeur titulaire et chef intérimaire du Département de pratique pharmaceutique de la Faculté de pharmacie de l'Université Purdue et professeur associé de l'École de médecine de I'Université d'Indiana, à Indianapolis, en Indiana, aux États-Unis. II est également rédacteur adjoint du JCPH.

Intérêts concurrents : aucun déclaré.

Adresse de correspondance :

Dr James E Tisdale

Department of Pharmacy Practice

College of Pharmacy

Purdue University

640 Eskenazi Ave

Indianapolis IN 46202 USA

Courriel : jtisdale@purdue.edu 